\title{
Direct evidence for structural origin of stress-induced magnetic anisotropy in $\mathrm{Fe}-\mathrm{Si}-\mathrm{B}-\mathrm{Nb}-\mathrm{Cu}$ nanocrystalline alloys
}

\author{
M. Ohnuma a) and K. Hono \\ National Institute for Materials Science, 1-2-1 Sengen, Tsukuba 305-0047, Japan \\ T. Yanai and H. Fukunaga \\ Nagasaki University, Nagasaki 852-8521, Japan \\ Y. Yoshizawa \\ Hitachi Metals Ltd., 5200 Mikajiri, Kumagaya, Saitama 360-0843, Japan
}

(Received 17 June 2003; accepted 4 August 2003)

\begin{abstract}
The structural origin of magnetic anisotropy in $\mathrm{Fe}-\mathrm{Si}-\mathrm{B}-\mathrm{Nb}-\mathrm{Cu}$ alloys annealed under a tensile stress of $200 \mathrm{MPa}$ is studied by transmission x-ray diffraction. The diffraction peak of the (310) plane, whose normal vector is parallel to the tensile direction (ribbon direction), appears at a lower angle than the one perpendicular to it by about $0.1^{\circ}$. This indicates that the spacing of the (310) plane normal to the tensile direction is about $0.2 \%$ larger than the one parallel to it. This is direct evidence for the structural origin of the stress-induced magnetic anisotropy of nanocrystalline soft magnetic alloys. (C) 2003 American Institute of Physics. [DOI: 10.1063/1.1615672]
\end{abstract}

$\mathrm{Fe}-\mathrm{Si}-\mathrm{B}-\mathrm{Nb}-\mathrm{Cu}$ nanocrystalline soft magnetic alloys (FINEMET®) are one of the materials that utilize unique properties by controlling the grain size to less than $20 \mathrm{~nm}$. Since the report by Yoshizawa et al. ${ }^{1}$ in 1988 , a number of investigations on nanocrystalline soft magnetic alloys have been carried out to optimize the properties that are suitable for specific applications leading to increase in saturation flux density, Curie temperature, and in-plane magnetic anisotropy. $^{2-4}$ The in-plane magnetic anisotropy is especially important for applications in high-frequency regions, because large magnetic anisotropy increases the magnetic resonance frequency and produces a flat permeability-frequency ( $\mu$-f) response. Magnetic field annealing is commercially used to induce magnetic anisotropy; however, it is necessary to substitute $\mathrm{Co}$ for Fe to obtain an anisotropy field that is larger than $1000 \mathrm{~A} / \mathrm{m}$ by this method. ${ }^{3,4}$ In contrast, the anisotropy field that is induced by stress annealing can exhibit larger values than $1000 \mathrm{~A} / \mathrm{m}$ without substituting Co for Fe. The stress-induced anisotropy of amorphous alloys annealed below crystallization temperatures $\left(300-400{ }^{\circ} \mathrm{C}\right.$ ) has been studied widely. ${ }^{5,6}$ Himovich et $a .^{6}{ }^{6}$ studied the structural origin of the anisotropy by using energy dispersive x-ray diffraction. They observed the difference in intensities between the two diffraction vectors (parallel and perpendicular to the stress direction) and attributed it to the anisotropy in the angular distribution of the atomic bonds in the amorphous structure. Kraus et al. ${ }^{7}$ reported that the stress-induced magnetic anisotropy that is larger than $1000 \mathrm{~A} / \mathrm{m}$ can be induced in FINEMET®-type alloys by crystallizing the amorphous precursor under a tensile stress. The subsequent studies showed that the anisotropy fields that are obtained by annealing under a tensile stress can become larger than $3000 \mathrm{~A} / \mathrm{m}$ depending on the tensile stress. ${ }^{8-12}$ However, the alloys do

a) Author to whom correspondence should be addressed at: Materials Engineering Laboratory, National Institute for Materials Science, 1-2-1 Sengen, Tsukuba 305-0047, Japan; electronic mail: ohnuma.masato@nims.go.jp not show any anisotropy in shape or texture. ${ }^{7,9}$ These nanocrystalline alloys consist of two phases, i.e., body-centeredcubic (bcc) crystals and a remaining amorphous phase, thus the origin of this stress-induced anisotropy is not clear. Kraus et $a .^{7}$ suggested that the anelastic polarization of the amorphous intergranular phase is mainly responsible for the anisotropy. Herzer ${ }^{8}$ pointed out a similarity of the dependences of stress-induced magnetic anisotropy and magnetostriction on the Si concentration and concluded that the anisotropy is attributed to the deformation of the crystalline phase due to the internal stress. Hoffmann et al. ${ }^{9}$ explained that the stressinduced anisotropy and the elongation of the samples by stress annealing is due to the pair ordering of Fe and $\mathrm{Si}$ in the framework of Herzer's model. However, no direct evidence for the structural origin of the stress-induced anisotropy in nanocrystalline soft magnetic alloys has been reported.

Fukunaga et al. ${ }^{10-12}$ made troidal cores using an alloy that was annealed under the stress and showed a flat $\mu$-f property up to $1 \mathrm{MHz}$. Its maximum dc-bias field was two times larger than that of the ferrite cut core. Therefore, understanding the origin of the stress-induced anisotropy is very important from both fundamental and technical points of view. The aim of this study is to clarify the mechanism of the magnetic anisotropy in the nanocrystalline alloys annealed under a tensile stress using a conventional x-ray diffraction (XRD) with a transmission mode. The merit of using XRD with the transmission mode is that the diffraction vector $q$ can be taken in a sample plane. We measured two profiles with different diffraction vectors, i.e., parallel and perpendicular to the ribbon direction (tensile stress direction) in each specimen.

The composition of the alloy ribbons by the single-roll melt-spinning method in this study was $\mathrm{Fe}_{73.5} \mathrm{Si}_{15.5} \mathrm{~B}_{7} \mathrm{Nb}_{3} \mathrm{Cu}_{1}$. The samples were quickly heated to $500{ }^{\circ} \mathrm{C}$, and a tensile stress of $200 \mathrm{MPa}$ was applied parallel to the ribbon direction. Then, the samples were heated to $570{ }^{\circ} \mathrm{C}$ at a heating rate of $7^{\circ} \mathrm{C} / \mathrm{min}$ and kept at $570^{\circ} \mathrm{C}$ for 


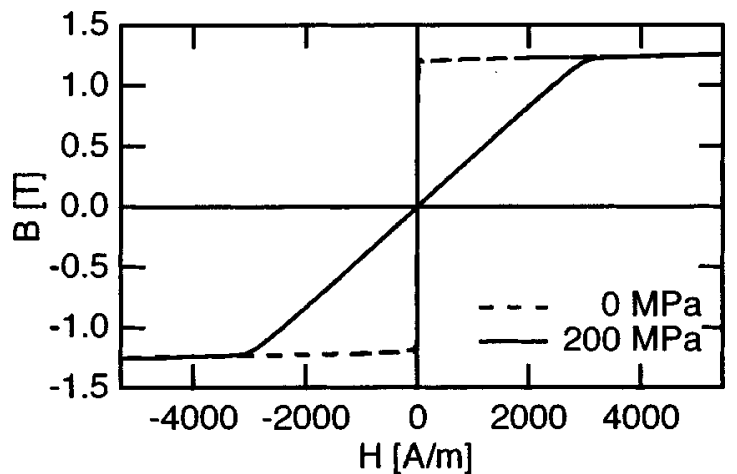

FIG. 1. Magnetization curves of $\mathrm{Fe}_{73.5} \mathrm{Si}_{15.5} \mathrm{~B}_{7} \mathrm{Nb}_{3} \mathrm{Cu}_{1}$ alloys annealed with and without the application of a tensile stress of $200 \mathrm{MPa}$. Both curves are measured along the ribbon direction (parallel to the tensile stress).

20 min under the tensile stress. The same annealing process without a tensile stress was applied to the same samples for comparison. The details of the sample preparation method are described in Refs. $10-12$

Figure 1 shows in-plane magnetization curves of $\mathrm{Fe}_{73.5} \mathrm{Si}_{15.5} \mathrm{~B}_{7} \mathrm{Nb}_{3} \mathrm{Cu}_{1}$ nanocrystalline alloys parallel to the ribbon direction with and without the tensile stress. The alloys annealed under the tensile stress show a large induced magnetic anisotropy field of $3000 \mathrm{~A} / \mathrm{m}$, while the alloy annealed without the tensile stress does not show any magnetic anisotropy. Small-angle x-ray scattering (SAXS) measure-
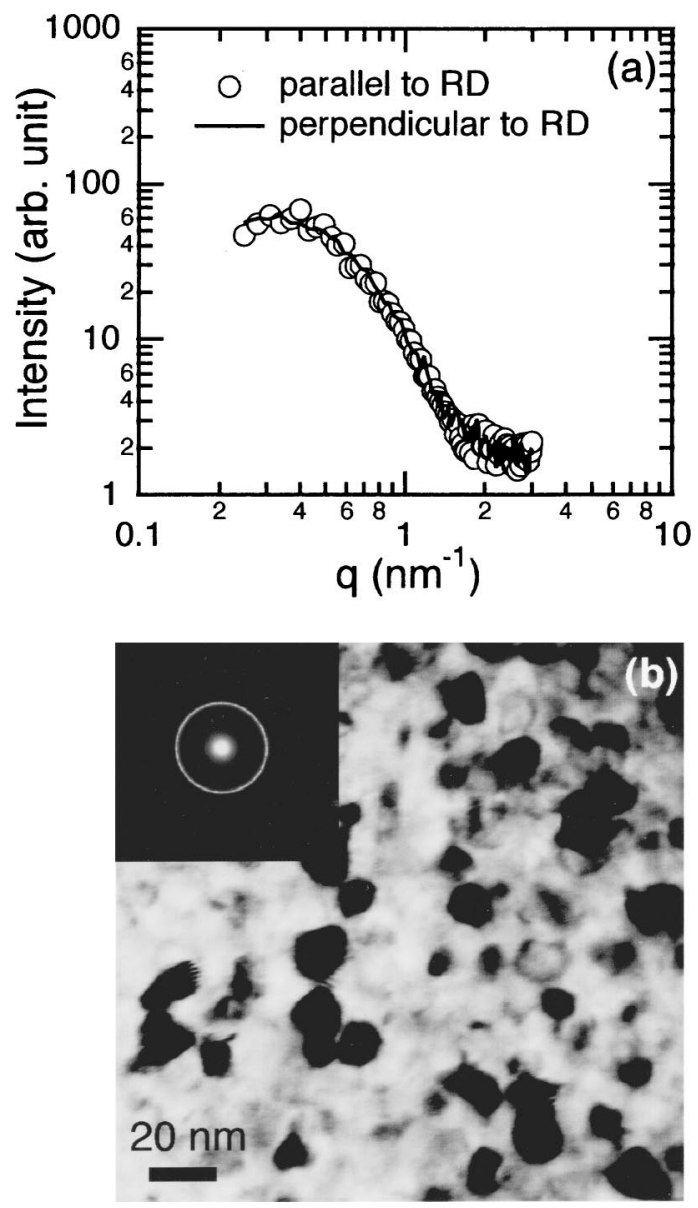

FIG. 2. (a) SAXS profiles and (b) TEM micrograph and SAED pattern of the $\mathrm{Fe}_{73.5} \mathrm{Si}_{15.5} \mathrm{~B}_{7} \mathrm{Nb}_{3} \mathrm{Cu}_{1}$ alloy annealed under a tensile stress of $200 \mathrm{MPa}$. Scattering vectors are parallel and perpendicular to the ribbon direction (RD) in (a). dicular to the tensile direction in the alloy annealed under the
(RD) in (a).
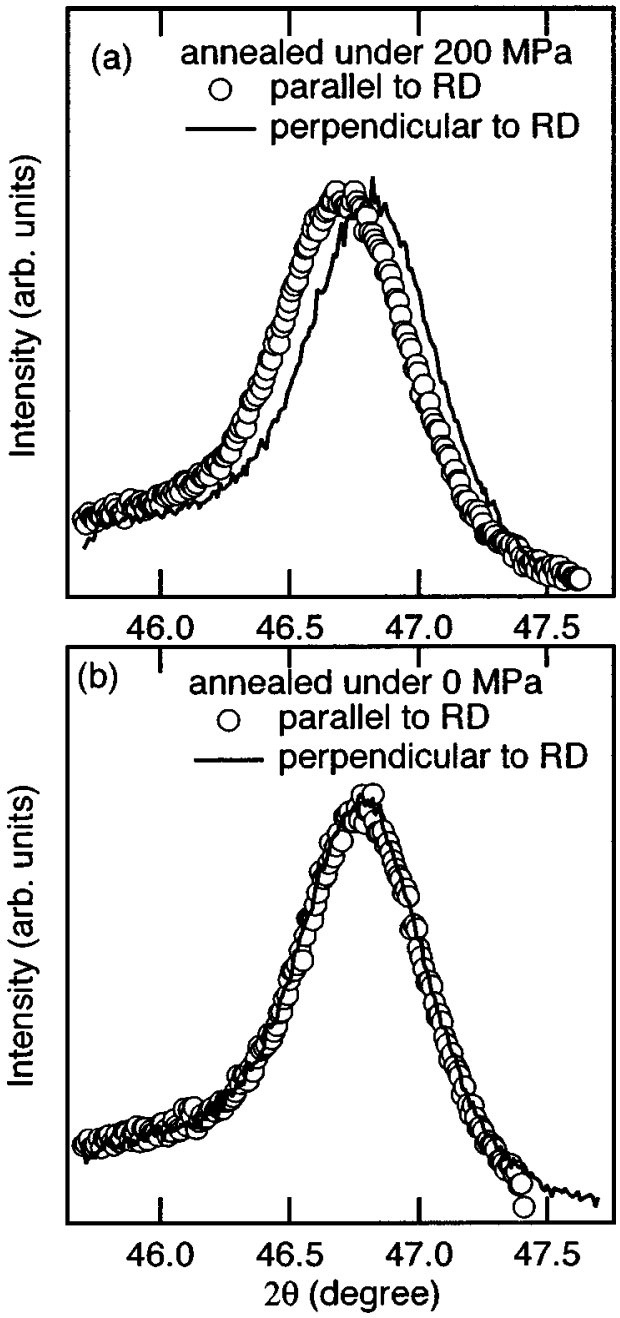

FIG. 3. XRD profiles with diffraction vectors are parallel and perpendicular to the $\mathrm{RD}$ of $\mathrm{Fe}_{73.5} \mathrm{Si}_{15.5} \mathrm{~B}_{7} \mathrm{Nb}_{3} \mathrm{Cu}_{1}$ alloys annealed (a) under a tensile stress of $200 \mathrm{MPa}$, (b) without applying the tensile stress.

ments and transmission electron microscope (TEM) observations, including selected area electron diffraction (SAED), were carried out on the sample annealed under the stress to check the shape of the anisotropy and its texture. As shown in Fig. 2, SAXS, TEM, and SAED show isotropic profiles (or shapes), indicating the absence of shape anisotropy and texture as described in the previous studies. ${ }^{7,9}$

In contrast, transmission XRD profiles of the alloy annealed under the tensile stress show a clear difference between the two directions. The measurements were performed using an instrument for conventional XRD with a Mo target, incident monochromator, and solid-state detector. By offsetting the sample stage $90^{\circ}$ from the reflection mode, we can select a diffraction vector at a certain direction in the sample plane using the conventional $\theta-2 \theta$ scan program. The samples were mounted on a glass substrate with a thickness of $0.5 \mathrm{~mm}$ to keep a high reproducibility of the sample position. Figure 3 shows XRD profiles of the alloys with and without the application of the tensile stress. To obtain a high accuracy and a reasonably high intensity, we chose the (310) peak for comparison in the two directions. The peak with a diffraction vector parallel to the tensile direction appears at a lower angle than the one with a diffraction vector perpen- 

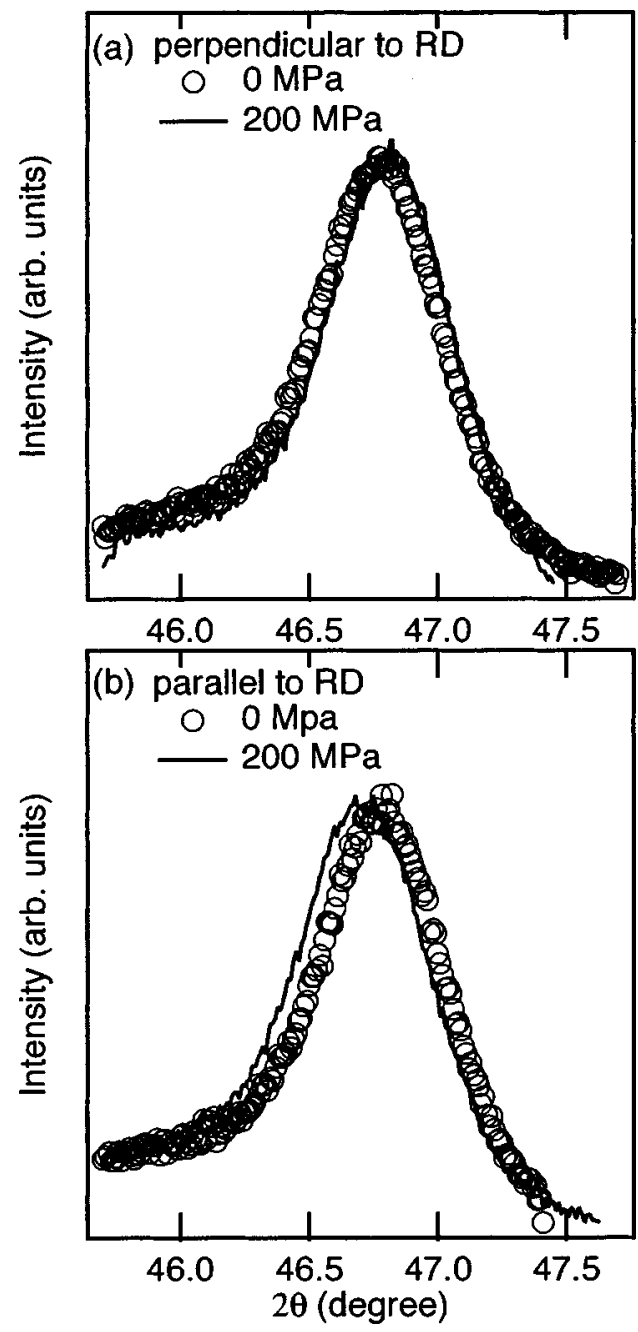

FIG. 4. XRD profiles of $\mathrm{Fe}_{73.5} \mathrm{Si}_{15.5} \mathrm{~B}_{7} \mathrm{Nb}_{3} \mathrm{Cu}_{1}$ alloys annealed with/without the application of the tensile stress, (a) perpendicular and (b) parallel to RD.

tensile stress. The difference of the peak top position between them is $0.1^{\circ}$ in $2 \theta$ as shown in Fig. 3(a), corresponding to the ratio of the difference in the distance of the plane, $\Delta d\left(=d_{\text {parallel }} 200 \mathrm{MPa}-d_{\text {perpend }} 200 \mathrm{MPa}\right) / d_{\text {perpend }} 200 \mathrm{MPa}$ of about $0.2 \%$. In contrast, the peak positions of these two directions in the alloy, annealed without the application of the tensile stress, match each other perfectly as shown in Fig. 3(b), indicating that there is no difference in the spacing of (310) between the two directions. Although there is a small difference in the perpendicular direction between the spacing of (310) in the alloy without the field, $d_{\text {perpend }} 0 \mathrm{MPa}$, and that of the alloy annealed under the stress, $d_{\text {perpend }}{ }^{200 \mathrm{MPa}}$, a much larger change occurs in the direction parallel to the ribbon direction, i.e., the tensile stress direction as shown in Fig. 4. These results show that the lattice spacing of the (310) plane is elongated by annealing under the tensile stress. This causes the distance of the Fe atoms to elongate along the direction parallel to the tensile stress. Consequently, the magnetic anisotropy will appear and the domain wall will array perpendicular to this elongated direction as shown in the previous work. ${ }^{7,9,10}$ Because the measured peaks are from the bcc-Fe-Si phase, the structural origin of the anisotropy is mainly in the crystalline phase, not in the amorphous phase as previously suggested by Kraus et al. ${ }^{7}$ The obtained value of the elongation parallel to the tensile stress almost agrees with the expected values based on both magnetoelastic and pair ordering models as described in the previous studies. ${ }^{8,9}$

In summary, we have detected a clear difference in the lattice spacing of the (310) planes between the two directions in the stress annealed $\mathrm{Fe}_{73.5} \mathrm{Si}_{15.5} \mathrm{~B}_{7} \mathrm{Nb}_{3} \mathrm{Cu}_{1}$ nanocrystalline soft magnetic alloys. Although it is not clear whether or not the detected difference is attributed to the directional pair ordered array induced by distortion or distortion by internal stress, the lattice spacing along the tensile stress is elongated in the alloy annealed under the tensile stress. No such structural anisotropy has been observed in the alloy annealed without a tensile stress. This is direct evidence for the structural origin of stress-induced magnetic anisotropy of nanocrystalline alloys.

This work was supported by the Special Coordination Funds for Promoting Science and Technology on "Nanohetero Metallic Materials" from the Ministry of Education, Sports, Culture, Science, and Technology of Japan.

${ }^{1}$ Y. Yoshizawa, S. Oguma, and K. Yamauchi, J. Appl. Phys. 64, 6044 (1988).

${ }^{2}$ M. A. Willard, M. Q. Huang, D. E. Laughlin, M. E. McHenry, J. O. Cross, and V. G. Harris, J. Appl. Phys. 85, 4421 (1999).

${ }^{3}$ Y. Yoshizawa, S. Fujii, D. H. Ping, M. Ohnuma, and K. Hono, Scr. Mater. 48, 863 (2003).

${ }^{4}$ M. Ohnuma, D. H. Ping, T. Abe, H. Onodera, K. Hono, and Y. Yoshizawa, J. Appl. Phys. 93, 9186 (2003).

${ }^{5}$ O. V. Nielsen, IEEE Trans. Magn. 5, 2008 (1985).

${ }^{6}$ J. Haimovich, T. Jagielinski, and T. Egami, J. Appl. Phys. 57, 3581 (1985).

${ }^{7}$ L. Kraus, K. Zaveta, O. Heczko, P. Duhaj, G. Vlasak, and T. Schnaider, J. Magn. Magn. Mater. 112, 275 (1992).

${ }^{8}$ G. Herzer, IEEE Trans. Magn. 30, 4800 (1994).

${ }^{9}$ B. Hofmann and H. Kronmüller, J. Magn. Magn. Mater. 152, 91 (1992).

${ }^{10}$ H. Fukunaga, T. Yanai, H. Tanaka, M. Nakano, K. Takahashi, and Y. Yoshizawa, IEEE Trans. Magn. 38, 1 (2002).

${ }^{11}$ H. Fukunaga, H. Tanaka, T. Yanai, M. Nakano, K. Takahashi, and Y. Yoshizawa, J. Magn. Magn. Mater. 242, 279 (2002).

${ }^{12} \mathrm{H}$. Fukunaga, T. Yanai, H. Tanaka, M. Nakano, K. Takahashi, and Y. Yoshizawa, J. Appl. Phys. 87, 7103 (2000). 